\title{
ÍNDICE ANEEL DE SATISFAÇÃO DO CONSUMIDOR, INDICADORES DE VALORAÇÃO E DESEMPENHO DE COMPANHIAS DISTRIBUIDORAS DE ENERGIA ELÉTRICA
}

\section{ANEEL CONSUMER SATISFACTION INDEX, VALUATION AND PERFORMANCE INDICATORS OF ELECTRICITY DISTRIBUTION COMPANIES}

\author{
CAROLINE SZPANICK DE OLIVEIRA \\ Universidade Federal do Paraná - UFPR \\ Especialista em Gestão Contábil e Tributária
}

Orcid: https://orcid.org/0000-0003-2163-0813 / E-mail: cah.szp@gmail.com

Av. Prefeito Lothário Meissner, 632 - Campus III - Jardim Botânico

CEP: $80210-170$ - Curitiba/PR - Brasil

IAGO FRANÇA LOPES

Universidade Federal do Paraná - UFPR

Doutorando no PPG em Contabilidade - UFPR

Orcid: https://orcid.org/0000-0001-7627-4815 / E-mail: iagofrancalopes@gmail.com

ALISON MARTINS MEURER

Universidade Federal do Paraná - UFPR

Doutorando no PPG em Contabilidade - UFPR

Orcid: https://orcid.org/0000-0002-3704-933X / E-mail: alisonmmeurer@gmail.com

\author{
CLAUDIO MARCELO EDWARDS BARROS \\ Professor na Universidade Federal do Paraná - UFPR \\ Doutor em Contabilidade
}

Orcid: https://orcid.org/0000-0001-7431-1627 / E-mail: claudiomedwards@hotmail.com

\section{RESUMO}

O objetivo do estudo é verificar a associação entre o Índice da Agência Nacional de Energia Elétrica (ANEEL) de Satisfação do Consumidor (IASC) e os indicadores de valoração e de desempenho de companhias distribuidoras de energia elétrica listadas na Brasil Bolsa Balcão [B] $]^{3}$. A amostra consistiu de 16 empresas distribuidoras de energia elétrica. Os dados referentes ao Índice ANEEL de Satisfação do Consumidor e valoração e desempenho das organizações foram obtidos por meio de fontes secundárias. A análise da correlação de Spearman indica que há associação positiva e significante entre o fator do IASC e o fator de desempenho. Outro resultado da pesquisa é que não há associação significante entre a qualidade dos serviços prestados e a valoração de mercado das distribuidoras de energia elétrica. Logo, os indicadores de qualidade podem traduzir a eficiência da organização em aproveitar seus recursos na prestação de serviços ao cliente, que por sua vez, se associaria ao desempenho financeiro. Por fim, ilustra-se que há correlação positiva e significante entre o fator de desempenho e o fator de valoração. Esta investigação contribui para o campo de pesquisa, à medida que reconhece a necessidade de integração de variáveis não quantitativas e quantitativas para o desenvolvimento das organizações, em especial os setores que recebem o intervencionismo do Estado de modo direto no que incumbe fomento, regulação e fiscalização.

Palavras-chave: ANEEL. Satisfação do consumidor. Valoração de empresa. Desempenho de companhias. Setor elétrico. 


\section{ABSTRACT}

The aim of the study is to verify the association between National Electric Energy Agency (ANEEL) Consumer Satisfaction Index (IASC), valuation and performance indicators of electricity distribution companies listed in Brazil Bolsa Balcão $[B]^{3}$. The sample consisted of 16 electricity distribution companies. Data regarding the ANEEL Index of Consumer Satisfaction and valuation and performance of organizations were obtained from secondary sources. Spearman correlation analysis indicates that there is a positive and significant association between the IASC factor and the performance factor. Another result of the research is that there is no significant association between the quality of the services provided and the market valuation of the electricity distributors. Thus, quality indicators can translate the organization's efficiency in leveraging its resources to provide customer services, which in turn would be associated with financial performance. Finally, it is illustrated that there is a positive and significant correlation between performance factor and valuation factor. This research contributes to the field of research, as it recognizes the need for integration of non-quantitative and quantitative variables for the development of organizations, especially sectors that receive state interventionism directly in terms of promotion, regulation and supervision.

Keywords: ANEEL. Consumer satisfaction. Company valuation. Company performance. Electric sector.

\section{INTRODUÇÃO}

Durante as décadas de 1990 e 2000 o setor elétrico brasileiro passou por um processo de reestruturação sustentado pela privatização de companhias elétricas, fim do subsídio cruzado, desverticalização do setor e incentivo a competição, tendo como foco o aumento da eficiência energética, a promoção da concorrência e a melhoria na qualidade dos serviços prestados (GUIMARÃES, 2015; WALVIS; GONÇALVES, 2014; GOLDENBERG; PRADO, 2003). A criação e operacionalização desse novo modelo estrutural para o setor foi amparado pela ANEEL.

A ANEEL foi criada a partir da Lei no 9.427 de 1996 (BRASIL, 1996), com o objetivo de fiscalizar e regular a geração, transmissão e distribuição de energia elétrica de acordo com as diretrizes do Governo Federal (ANEEL, 2018). Entre as ações realizadas com intuito de maximizar a qualidade da distribuição da energia elétrica tem-se a avaliação dos indicadores da ANEEL, voltados para a satisfação dos serviços prestados, duração e frequência do fornecimento elétrico e no atendimento a ocorrências emergenciais (ALMEIDA et al., 2010).

Referente a avaliação da satisfação dos serviços prestados, a ANEEL possui o Índice ANEEL de Satisfação do Consumidor (IASC), que avalia desde o ano 2000 a qualidade e o valor percebido pelos consumidores referente a relação custo-benefício, a satisfação global, a confiança no fornecedor e a fidelidade dos consumidores em relação as empresas distribuidoras de energia elétrica. Esta iniciativa tem como finalidade aprimorar a regulação e a fiscalização das ações promovidas pela agência (ANEEL, 2015, 2016).

Assim, observar a qualidade dos serviços prestados consubstanciada na satisfação dos consumidores é relevante não somente sob o enfoque operacional, mas também porque "existe uma crença [...] de que a melhoria da qualidade pode alavancar a posição competitiva e financeira da empresa" (ALBUQUERQUE; CARVALHO; BONIZIO, 2014, p. 140). Quando a melhoria da qualidade reflete na posição competitiva da empresa, pode-se 
observar por meio do comportamento dos indicadores de valoração esse processo. Tais indicadores são comumente inscritos no campo empírico por meio do $Q$ de Tobin, no Valor da Firma (SANTANA; LIMA, 2004) e na Capitalização do Mercado (BODIE; KANE; MARCUS, 2014; ROSTAGNO; SOARES; SOARES, 2006).

Transpor essas métricas do campo de finanças para identificação de possíveis associações com elementos qualitativos do setor elétrico, por meio do IASC mostra-se oportuno, à medida que tal setor recebe elevada atenção dos pesquisadores, mas ainda representa um campo de pesquisa esparso (GASPARETTO et al., 2018). Outro ponto é o fato da pesquisa fornecer ao campo empírico como o setor elétrico responde em termos de comportamento do mercado acionário frente a dependência da satisfação do consumidor final.

O que chama atenção em termos de desenvolvimento empírico é que "altos níveis de satisfação se correlacionam com altos níveis de desempenho" (CATAPAN et al., 2017, p. 1). Fato que se mostra como uma oportunidade de pesquisa, principalmente restringindo-se a um setor que recebe elevada interferência do Estado. Dessa forma, recorrendo ao campo empírico no Brasil percebe-se que os estudos que abordam a qualidade dos serviços e suas consequências sobre o desempenho financeiro no setor elétrico ainda são precursores e têm apresentado resultados divergentes (RESENDE; CARDOSO, 2019; CATAPAN et al., 2017; MARINHO; RESENDE, 2016; ALMEIDA et al., 2010).

Almeida et al. (2010) encontraram evidências de relação da satisfação dos consumidores com a rentabilidade de empresas distribuidoras de energia elétrica. Por outro lado, não foi possível afirmar que há diferenças nas variáveis de desempenho financeiro entre empresas com melhor e pior qualidade dos serviços prestados. O cenário de evidências científicas apresenta-se plural (RESENDE; CARDOSO, 2019; ALMEIDA et al., 2010), fato que incentiva a investigação da associação entre o Índice ANEEL de Satisfação do Consumidor e desempenho de companhias elétricas. Dessa forma, esta pesquisa busca responder a seguinte questão: Qual a associação entre o Índice ANEEL de Satisfação do Consumidor, para os indicadores de valoração e de desempenho de companhias distribuidoras de energia elétrica listadas na Brasil Bolsa Balcão $[B]^{3}$ ? Consequentemente, tem-se por objetivo verificar a associação entre o Índice ANEEL de Satisfação do Consumidor, para os indicadores de valoração e de desempenho de companhias distribuidoras de energia elétrica listadas na Brasil Bolsa Balcão [B] $]^{3}$.

As empresas de distribuição de energia elétrica possuem relacionamento com o último consumidor da cadeia de fornecimento, tanto na demanda de energia consumida, quanto ao inadimplemento. Além disso, o setor elétrico afeta todos os agentes econômicos do país e a sociedade como um todo, sendo perspicaz analisar o relacionamento do índice de satisfação dos clientes dessas companhias com o seu desempenho. Assim, sob a pespectiva prática busca-se apresentar para os usuários o possível relacionamento entre o Índice ANEEL de Satisfação do Consumidor, indicadores de valoração e de desempenho e consequentemente incentivar a organização a aperfeiçoar os serviços prestados. Já sob o ponto de vista dos investidores e das organizações, a relevância do estudo paira em identificar se o Índice ANEEL de Satisfação do Consumidor contribui com a geração de valor empresarial com base em indicadores, sejam de desempenho e/ou valoração.

Para as organizações, compreender a associação da qualidade dos serviços prestados, por meio da satisfação dos consumidores, com a valoração e o desempenho pode auxiliar no desenvolvimento de ações que maximizem a eficiência da organização. Nesse sentido, com a existência de associação entre estas variáveis haverá indicação de que a qualidade dos 
serviços prestados transcende as impressões dos consumidores sobre a organização, mas também se correlaciona com os resultados que afetam diretamente a capacidade de obtenção de desempenhos financeiros e econômicos satisfatórios. Assim, medidas organizacionais poderão a longo prazo ser adotadas no que tange maximazar a satisfação do consumidor e consequentemente o valor da empresa no mercado tenderá a aumentar.

O estudo contribui para a ampliação das discussões acerca das relações existentes entre a qualidade, desempenho financeiro e valoração de mercado, à medida que comumente estas variáveis são analisadas de forma isolada. Amplia-se também os achados sobre o setor de energia elétrica, visto que a literatura tem-se mostrado plural e com resultados que merecem ainda mais atenção de pesquisadores, com vistas a enriquecer o mapeamento de achados que melhor contribuam com os usuários da informação contábil em termos quantitativos e qualitativos (RESENDE; CARDOSO, 2019; CATAPAN et al., 2017; MARINHO; RESENDE, 2016; ALBUQUERQUE et al., 2014; ALMEIDA et al., 2010).

\section{REFERENCIAL TEÓRICO}

Para sustentar em termos teóricos esta investigação, constrói-se evidências relacionadas ao índice ANEEL de satisfação do consumidor e referente aos antecedentes empíricos para o desenvolvimento das hipóteses de pesquisa.

\section{1 ÍNDICE ANEEL DE SATISFAÇÃO DO CONSUMIDOR}

Para Perez (2017) a implantação de um sistema de distribuição de energia elétrica impacta a vida daqueles que passam a fazer uso deste sistema. A dependência deste tipo de serviço coloca em voga a necessidade de controles dos órgãos reguladores sobre a qualidade dos serviços atrelados a distribuição de energia elétrica. O marco regulatório do setor elétrico subdivide tal qualidade em dimensões, sendo: (i) qualidade dos serviços prestados, em que se avalia a distribuição de energia elétrica; (ii) qualidade do produto que abrange elementos da energia elétrica, como tensão e regimes permanentes; e (iii) as atividades comerciais das distribuidoras de energia elétrica, em termos de cumprimento de prazos, cobranças, contratos e demais elementos que perpassam este tipo de atividade (PEREZ, 2017).

A ANEEL exige que as distribuidoras de energia elétrica disponham de "[...] equipes capacitadas e quantidade de medidores compatíveis com o número de reclamações associadas à qualidade do produto" (ANEEL, 2017, p. 32), bem como o acompanhamento de indicadores específicos para avaliar a frequência e duração do fornecimento de energia elétrica (ANEEL, 2017). Anualmente a ANEEL avalia a satisfação do consumidor residencial em relação aos serviços de distribuição de energia elétrica de todo o país, como forma de mensurar a qualidade percebida dos serviços de energia elétrica.

Essa análise é realizada por meio do Índice ANEEL de Satisfação do Consumidor (IASC). Para construir o índice, anualmente são realizadas aproximadamente vinte e cinco mil entrevistas com consumidores de todas as distribuidoras, concessionárias e permissionárias do Brasil. As empresas que tiverem melhor avaliação pelos consumidores, ou seja, cujo índice seja maior, são premiadas com troféu, certificado e selo IASC (ANEEL, 2015). A Figura 1 apresenta os elementos considerados pelo Índice ANEEL de Satisfação do Consumidor. 
Figura 1 - Variáveis do IASC.

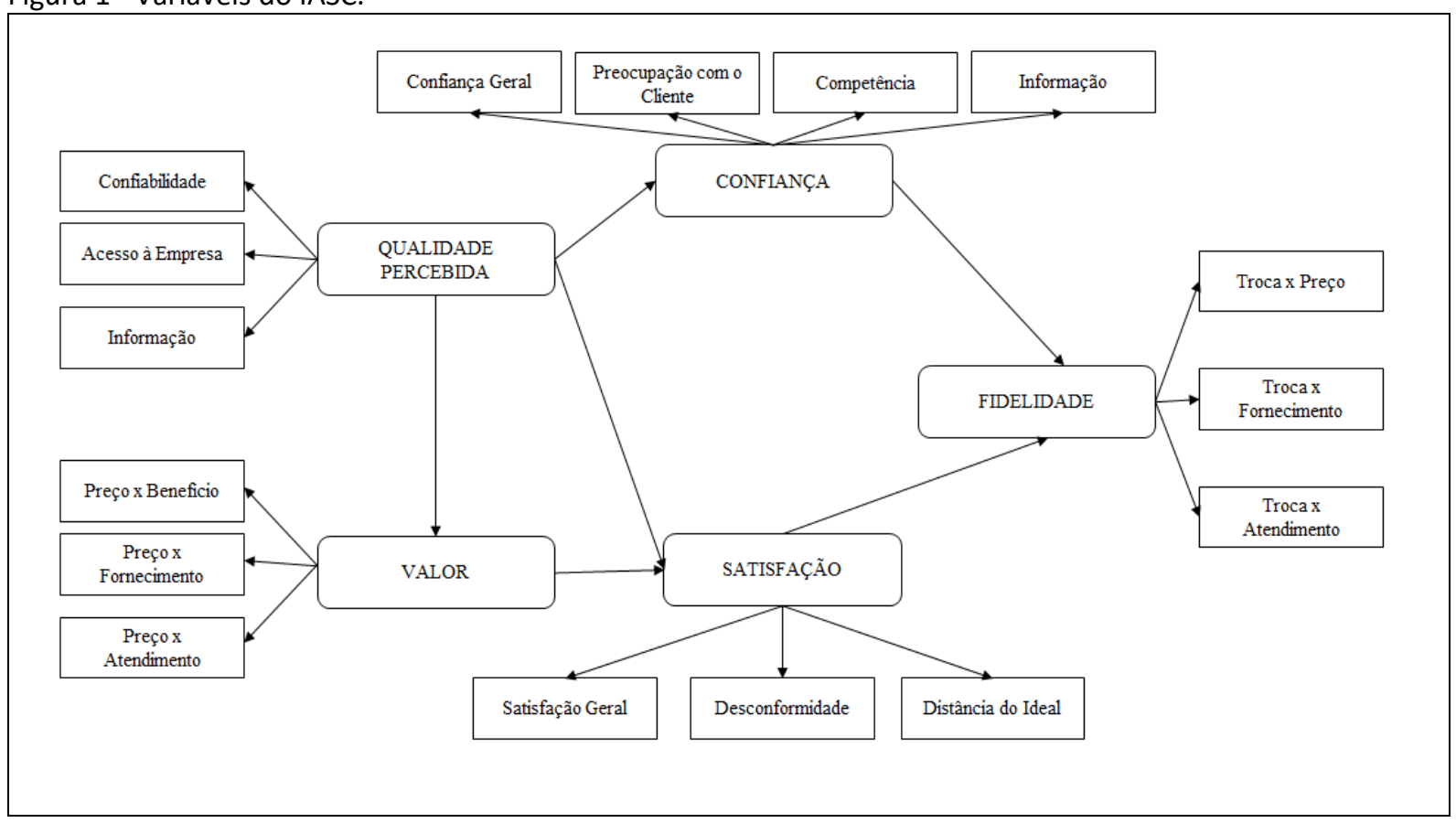

Fonte: Relatório Pesquisa IASC (2017).

A partir da Figura 1 nota-se que a 'Qualidade Percebida' é avaliada a partir de três itens. Estes itens foram definidos por meio de pesquisas qualitativas realizadas no ano 2000 e consolidados por representantes da ANEEL, como itens que versam sobre informações ao cliente, acesso a empresa e confiabilidade nos serviços prestados. O 'Valor' aborda aspectos econômicos da percepção do consumidor. A 'Satisfação' mensura a satisfação global dos clientes em relação às três dimensões, a saber: satisfação global, distância para empresa ideal e desconformidade global. A 'Lealdade' mede a confiança dos consumidores em relação ao seu fornecedor de energia elétrica. A 'Fidelidade' avalia a possibilidade de troca do fornecedor de energia elétrica devido ao valor tarifário, fornecimento e/ou atendimento da empresa. (ALMEIDA et al., 2010; ANEEL, 2017).

Mensurar a qualidade dos serviços de distribuição de energia elétrica é uma ação essencial e estratégica em termos organizacionais, à medida que este elemento aliado ao bom funcionamento e a confiabilidade do fornecimento de energia elétrica, por parte dos consumidores, pode ser um fator relevante diante de um mercado competitivo e interferir no desempenho da organização (LIMA, 2013).

\subsection{ANTECEDENTES EMPÍRICOS E DESENVOLVIMENTO DAS HIPÓTESES DE PESQUISA}

Estudos direcionam atenção para fomentar discussões acerca da qualidade e aspectos financeiros das distribuidoras de energia elétrica (MARINHO; RESENDE, 2016; ALBUQUERQUE et al., 2014; ALMEIDA et al., 2010; PIGNANELLI; CSILLAG, 2008). Pignanelli e Csillag (2008) buscaram analisar o efeito da qualidade no desempenho de 33 empresas participantes do Prêmio Nacional da Qualidade (PNQ), incluindo empresas distribuidoras de energia elétrica. O desempenho compreendeu variáveis de crescimento, lucratividade e valor de mercado. O período analisado foi de 20 anos, sendo de 1986 a 2005. Os resultados mostram que o desempenho de empresas que promoveram a adoção de técnicas de qualidade se mostrou maior que a média do setor no qual a organização está inserida. 0 
nível de crescimento e o valor de mercado não se diferenciou entre empresas que adotam uma gestão sob a perspectiva da qualidade.

Almeida et al. (2010) analisaram dados de 20 distribuidoras de energia elétrica de 2005 a 2009, a fim de descobrir se indicadores de qualidade interna dos serviços de distribuição de energia elétrica, como Duração Equivalente de Interrupção por Unidade Consumidora (DEC) e Freqüência Equivalente de Interrupção por Unidade Consumidora (FEC), se relacionam com o IASC e como essas variáveis afetam o desempenho financeiro das empresas, em termos de retorno sobre o ativo (ROA). A análise foi realizada por meio da técnica de regressão com o Método dos Mínimos Quadrados Ordinários (MQO). Os resultados indicaram haver relação negativa entre o DEC e o IASC. Além disso, foi identificada relação positiva e significante entre o ROA e o IASC, indicando que quanto maior a satisfação do cliente, melhor tende a ser os retorno sobre o ativo da distribuidora de energia elétrica. Tal processo indica que, quando as organizações atentam-se as necessidades dos seus clientes há impacto no desempenho das empresas do setor elétrico.

Albuquerque et al. (2014) verificaram se havia diferença na rentabilidade de acordo com a qualidade do serviço de 25 empresas de distribuição de energia elétrica. A qualidade do serviço prestado foi avaliada a partir dos indicadores DEC e FEC, já a rentabilidade foi operacionalizada pelo retorno sobre o ativo (ROA) e retorno sobre o investimento (ROI). Os dados compreenderam o período de 2001 a 2006, sendo analisados por meio de teste $t$. Os achados não puderam comprovar que há diferenças significantes na rentabilidade das empresas analisadas conforme os indicadores de qualidade do serviço prestado.

Marinho e Resende (2016) utilizaram a Análise Envoltória de Dados (Data Envelopment Analysis - DEA) para analisar a qualidade do seviço de distribuição de energia elétrica no Brasil entre 2010 a 2014. Foram analisadas 42 empresas e adotaram como input cinco indicadores obtidos da Superintendência de Regulação dos Serviços de Distribuição e como output o Índice ANEEL de Satisfação do Consumidor. Os resultados da pesquisa indicam que não é possivel rejeitar a hipótese de que houve mudança na qualidade dos serviços prestados no período analisado. Ademais, ao verificar os intervalos de confiança destaca-se ainda que a produtividade diminuiu em 16 empresas e aumentou em nove (MARINHO; RESENDE, 2016).

Percebe-se que as evidências empíricas apresentam diferentes constatações acerca da qualidade e desempenho financeiro das distribuidoras de energia elétrica. Assim, almejase contribuir e ampliar as discussões sobre a temática por meio da proposição das seguintes hipóteses de pesquisa:

$H_{1}$ : Existe associação entre o Índice ANEEL de Satisfação do Consumidor e os indicadores de valoração de empresas distribuidoras de energia elétrica listadas na $[B]^{3}$.

$\mathrm{H}_{2}$ : Existe associação entre o Índice ANEEL de Satisfação do Consumidor, indicadores de desempenho de empresas distribuidoras de energia elétrica listadas na $[B]^{3}$.

Não rejeitar essas hipóteses sinalizam para as empresas distribuidoras de energia elétrica listadas na $[B]^{3}$ a necessidade de considerar cada vez mais medidas não financeiras em conjunto com as medidas de valoração e de desempenho, ou seja, tende-se a capacidade de identificar uma relação de via dupla, a qual aponta que a posição dos consumidores quanto a satisfação dos serviços prestados alinha-se a performance da empresa.

\section{PROCEDIMENTOS METODOLÓGICOS}

Os procedimentos metodológicos consistem em descrever as técnicas adotadas para a construção de evidências que permitam discutir a associação entre o Índice da Agência

RGO - Revista Gestão Organizacional, Chapecó, v. 13, n. 2, p. 104-122, maio/ago. 2020. 
Nacional de Energia Elétrica (ANEEL) de Satisfação do Consumidor (IASC) e os indicadores de valoração e de desempenho de companhias distribuidoras de energia elétrica listadas na Brasil Bolsa Balcão $[\mathrm{B}]^{3}$.

\subsection{DELINEAMENTO DA PESQUISA}

Esta pesquisa possui características descritiva com abordagem quantitativa. Os dados utilizados na obtenção das evidências empíricas foram extraídos dos Relatórios de Pesquisa IASC e da plataforma Bloomberg ${ }^{\circledR}$. A população do estudo compreende as empresas distribuidoras de energia elétrica e como amostra, as empresas listadas na [B] ${ }^{3}$ que divulgaram dados entre os anos de 2010 a 2016. Exceto o ano de 2011 não foi investigado em virtude da não validade dos dados divulgados pela empresa vencedora da licitação no período para a realização da pesquisa junto aos consumidores (ANEEL, 2018). Assim, a composição da amostra da pesquisa é exposta na Tabela 1.

Tabela 1 - Composição da Amostra da Pesquisa

\begin{tabular}{lc}
\hline Empresas do Setor Elétrico & 33 \\
\hline (-) Empresas não avaliadas pelo IASC & $(14)$ \\
\hline (-) Empresas sem os dados para desempenho e valoração & $(03)$ \\
\hline (=) Empresas distribuidoras de energia elétrica aptas a participar & $\mathbf{1 6}$ \\
\hline
\end{tabular}

Fonte: elaborado pelos autores.

Os sete anos que englobam a pesquisa totalizam 99 observações. Da população de 33 empresas, 14 não possuíam dados referente ao Índice ANEEL de Satisfação do Consumidor, por caracterizarem-se a partir de atividade diferente à de distribuição de energia, como transmissão, geração e holdings. As empresas Energisa Mato Grosso - Distribuidora de Energia S. A., Cia Estadual de Distribuição de Energia Elétrica e Elektro Eletricidade e Serviços S. A. também foram excluídas da amostra por não possuírem dados na plataforma Bloomberg ${ }^{\circledR}$. Assim, a amostra final da pesquisa constitui-se de 16 empresas.

\subsection{OPERACIONALIZAÇÃO E COLETA DAS VARIÁVEIS}

Em termos operacionais, as variáveis que compõem a pesquisa estão dispostas no Quadro 1. Assim, apresentam-se as variáveis que corporizam o Índice ANEEL de Satisfação do Consumidor, aos indicadores de valoração e de desempenho.

As variáveis de índices amplos do setor elétrico (X4, X5, X6 e X7) compõem o Índice ANEEL de Satisfação do Consumidor (IASC). As variáveis X1, X2 e X3 compõem a variável de "Qualidade Percebida", sendo analisadas de forma individual para verificar o comportamento perante a formação fatorial. Já as variáveis referentes aos indicadores de valoração permitem comparar o valor de mercado com geração de caixa e custo de reposição dos investimentos. São considerados o $Q$ de Tobin, o Enterprise Value/Ebitda e a Capitação de Mercado. Por fim, as varíaveis de desempenho permitem avaliar o retorno aos acionistas e a rentabilidade das empresas, visto que são consideradas nesta dimensão o Ebitda/Receita, ROE, ROA, GC/AT e EVA. O Índice ANEEL de Satisfação do Consumidor foi obtido por meio de relatórios divulgados pela própria reguladora. Já os dados de valoração e desempenho das organizações foram obtidos por meio da plataforma Bloomberg ${ }^{\circledR}$. 
Quadro 1 - Variáveis da Pesquisa

\begin{tabular}{|c|c|c|c|c|}
\hline \multicolumn{2}{|c|}{ Variável } & $\begin{array}{l}\text { Identificação } \\
\text { da variável }\end{array}$ & Descrição & Referências \\
\hline \multirow{7}{*}{ 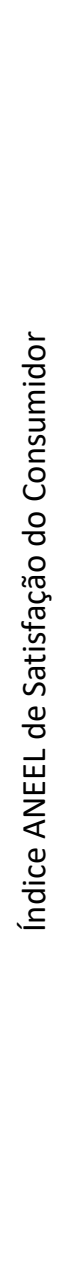 } & $\begin{array}{l}\text { Escore de } \\
\text { informação } \\
\text { ao cliente }\end{array}$ & $X_{1}$ & $\begin{array}{l}\text { Avalia a explicação sobre o uso adequado } \\
\text { da energia; segurança no valor cobrado; } \\
\text { atendimento igualitário aos consumidores; } \\
\text { informação/orientação sobre direitos e } \\
\text { deveres; e detalhamento das contas }\end{array}$ & \multirow{7}{*}{ ANEEL (2017) } \\
\hline & $\begin{array}{l}\text { Escore de } \\
\text { acesso à } \\
\text { empresa }\end{array}$ & $x_{2}$ & $\begin{array}{l}\text { Avalia a pontualidade na prestação de } \\
\text { serviços; facilidade para entrar em contato } \\
\text { com a empresa; cordialidade no } \\
\text { atendimento; facilidade de acesso aos } \\
\text { locais/meios de pagamento da conta; e } \\
\text { respostas rápidas às solicitações }\end{array}$ & \\
\hline & $\begin{array}{l}\text { Escore de } \\
\text { confiabilida } \\
\text { de nos } \\
\text { serviços }\end{array}$ & $X_{3}$ & $\begin{array}{c}\text { Avalia o fornecimento de energia sem } \\
\text { interrupção; fornecimento de energia sem } \\
\text { variação na tensão; avisos antecipados } \\
\text { sobre o corte de energia; confiabilidade } \\
\text { das soluções dadas; rapidez na volta da } \\
\text { energia; avisos antecipados sobre a falta } \\
\text { energia }\end{array}$ & \\
\hline & Valor & $\mathrm{X}_{4}$ & $\begin{array}{l}\text { Avalia a percepção do consumidor na } \\
\text { dimensão econômica }\end{array}$ & \\
\hline & Satisfação & $X_{5}$ & $\begin{array}{l}\text { Avalia a satisfação sobre a qualidade dos } \\
\text { serviços da concessionária ou } \\
\text { permissionária em relação à satisfação } \\
\text { global, distância para a empresa ideal e } \\
\text { desconformidade global }\end{array}$ & \\
\hline & Confiança & $X_{6}$ & $\begin{array}{c}\text { Avalia a confiança que os consumidores } \\
\text { possuem no seu fornecedor de energia } \\
\text { elétrica }\end{array}$ & \\
\hline & Fidelidade & $X_{7}$ & $\begin{array}{c}\text { Mede a intenção de troca de fornecedor } \\
\text { de energia elétrica }\end{array}$ & \\
\hline \multirow{3}{*}{ 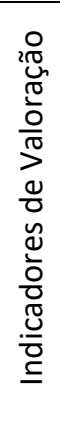 } & Q de Tobin & $\mathrm{V}_{1}$ & $\begin{array}{l}\text { Valor de mercado de ativos / Custo de } \\
\text { reposição dos ativos }\end{array}$ & $\begin{array}{c}\text { Famá e Barros } \\
(2000)\end{array}$ \\
\hline & $\begin{array}{l}\text { Enterprise } \\
\text { Value/Ebit } \\
\text { da }\end{array}$ & $V_{2}$ & $\begin{array}{l}\text { (Valor de mercado + Dívidas - Caixa e } \\
\text { equivalentes de caixa) / Ebitda }\end{array}$ & $\begin{array}{l}\text { Santana e Lima } \\
\qquad(2004)\end{array}$ \\
\hline & $\begin{array}{l}\text { Capitalizaç } \\
\text { ão de } \\
\text { Mercado }\end{array}$ & $V_{3}$ & $\begin{array}{l}\text { Preço de fechamento no mês da ação x no } \\
\text { de ações em circulação }\end{array}$ & $\begin{array}{l}\text { Bodie, Kane e } \\
\text { Marcus (2014) e } \\
\text { Rostagno, } \\
\text { Soares e Soares } \\
\text { (2006) }\end{array}$ \\
\hline \multirow{5}{*}{ 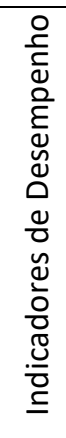 } & $\begin{array}{l}\text { Ebitda / } \\
\text { Receita } \\
\text { (Margem } \\
\text { Ebitda) }\end{array}$ & $D_{1}$ & $\begin{array}{c}\text { (Lucro operacional líquido + Depreciação + } \\
\text { Amortização) / Receita líquida }\end{array}$ & $\begin{array}{l}\text { Martins (1998) e } \\
\text { Neves Junior e } \\
\text { Batista (2005) }\end{array}$ \\
\hline & ROE & $D_{2}$ & Lucro líquido / Patrimônio líquido & \multirow{2}{*}{$\begin{array}{c}\text { Assaf Neto } \\
(2008)\end{array}$} \\
\hline & ROA & $\mathrm{D}_{3}$ & Lucro líquido / Ativo total & \\
\hline & GC / AT & $\mathrm{D}_{4}$ & Ebitda / Ativo Operacional & $\begin{array}{c}\text { Santana e Lima } \\
\text { (2004) }\end{array}$ \\
\hline & EVA & $D_{5}$ & $\begin{array}{c}\text { Lucro operacional - (Custo de } \\
\text { oportunidade total x Capital total) }\end{array}$ & $\begin{array}{c}\text { Assaf Neto } \\
(2008)\end{array}$ \\
\hline
\end{tabular}

Fonte: elaborado pelos autores. 


\subsection{PROCEDIMENTO DE ANÁLISE DOS DADOS}

Em termos de análise, é empregada a Análise Fatorial Exploratória com o objetivo de identificar os fatores que compõem o IASC, os indicadores de valoração e de desempenho. Em seguida é utilizada a correlação de Spearman com o intuito de analisar as associações existentes entre os fatores. O Quadro 2 apresenta o protocolo de análise dos dados.

Quadro 2 - Protocolo de análise dos dados.

\begin{tabular}{|c|c|c|c|}
\hline Indicadores & Objetivo & Parâmetro & Referência \\
\hline \multicolumn{4}{|c|}{ Estatística Descritiva } \\
\hline $\begin{array}{l}\text { Máximo, Mínimo, } \\
\text { Média, Mediana, } \\
\text { Desvio Padrão e } \\
\text { Coeficiente de } \\
\text { Variação }\end{array}$ & $\begin{array}{l}\text { Detalhar os dados que } \\
\text { compõem as variáveis } \\
\text { exploradas. }\end{array}$ & Não há. & $\begin{array}{c}\text { Fávero e } \\
\text { Belfiore (2017) }\end{array}$ \\
\hline \multicolumn{4}{|c|}{ Análise Fatorial Exploratória } \\
\hline $\begin{array}{l}\text { Matriz de correlação } \\
\text { de Pearson }\end{array}$ & $\begin{array}{l}\text { Analisar a correlação entre as } \\
\text { variáveis. }\end{array}$ & $\begin{array}{c}\text { Acima de } 0,30 \text { e } p \text { - } \\
\text { value }<0,05\end{array}$ & $\begin{array}{c}\text { Fávero e } \\
\text { Belfiore (2017) }\end{array}$ \\
\hline $\begin{array}{l}\text { Medida de } \\
\text { Adequação Amostral } \\
\text { (MAS) }\end{array}$ & $\begin{array}{ll}\text { Quantificar } & \text { as } \\
\text { intercorrelações entre } & \text { as } \\
\text { variáveis. } & \end{array}$ & $\begin{array}{c}<0,50: \text { Inaceitável } \\
\geq 0,50<0,60: \text { Ruim } \\
\geq 0,60<0,70: \\
\text { Medíocre } \\
\geq 0,70<0,80: \\
\text { Mediano } \\
>0,80: \text { Admirável } \\
\end{array}$ & $\begin{array}{l}\text { Hair Jr et al. } \\
\qquad(2009)\end{array}$ \\
\hline KMO & $\begin{array}{l}\text { Analisar a adequação global } \\
\text { com intuito de propiciar a } \\
\text { extração dos fatores. }\end{array}$ & $\begin{array}{c}\text { < 0,50: Inaceitável } \\
\geq 0,50<0,60: \text { Má } \\
\geq 0,60<0,70: \\
\text { Razoável } \\
\geq 0,70<0,80: \text { Média } \\
\geq 0,80<0,90: \text { Boa } \\
>\text { 0,90: Muito Boa }\end{array}$ & $\begin{array}{c}\text { Fávero e } \\
\text { Belfiore (2017) }\end{array}$ \\
\hline Teste de Bartlett & $\begin{array}{l}\text { Identificar a presença de } \\
\text { correlações } \\
\text { entre as variáveis. }\end{array}$ & $p$-value $<0,050$ & $\begin{array}{l}\text { Field (2009) e } \\
\text { Hair Jr et al. } \\
\text { (2009) }\end{array}$ \\
\hline \multicolumn{4}{|c|}{ Testes de Normalidade e Homogeneidade da Variância } \\
\hline Kolmogorov-Smirnov & $\begin{array}{l}\text { Verificar a normalidade dos } \\
\text { dados. }\end{array}$ & $p$-value $>0,050$ & Field (2009) \\
\hline \multicolumn{4}{|c|}{ Correlação de Spearman } \\
\hline Rho de Spearman & $\begin{array}{l}\text { Identificar a correlação } \\
\text { existente entre as variáveis } \\
\text { analisadas. }\end{array}$ & $\begin{array}{c}\text { A correlação será } \\
\text { verificada pelo } R h o \text { de } \\
\text { Spearman e a } \\
\text { siginificância pelo } p \text { - } \\
\text { value }<0,05\end{array}$ & $\begin{array}{c}\text { Fávero e } \\
\text { Belfiore (2017) }\end{array}$ \\
\hline
\end{tabular}

Fonte: elaborado pelos autores.

Os dados foram organizados no software Microsof $E_{x c e}{ }^{\circledR}$ e a análise dos dados foi realizada com o auxílio do software IBM SPSS Statistics $21^{\circledR}$. 


\section{ANÁLISE E DISCUSSÃO DOS RESULTADOS}

Para a articulação dos aspectos empíricos contruídos nesta investigação com os resultados já dispostos na literatura e análise das hipóteses, faz-se uso da estatística descritiva, análise fatorial exploratória e correlações de Spearman.

\subsection{ESTATÍSTICA DESCRITIVA}

Para verificar a dispersão das variáveis fez-se uso de estatística descritiva. Segundo Fávero e Belfiore (2017) ao observar as medidas descritivas o pesquisador consegue ter compreensão da forma com que os dados se comportam e é possível identificar valores atípicos e tendências. Na Tabela 2 é apresentada a estatística descritiva das variáveis exploradas nesta pesquisa.

Tabela 2 - Estatística descritiva

\begin{tabular}{|c|c|c|c|c|c|c|c|c|}
\hline \multicolumn{3}{|r|}{ Variáveis } & Máx. & Mín. & Média & Mediana & $\begin{array}{l}\text { Desvio } \\
\text { Padrão }\end{array}$ & $\begin{array}{l}\text { Coef. de } \\
\text { Variação }\end{array}$ \\
\hline \multirow{7}{*}{ I } & $X_{1}$ & Escore informação ao cliente & 0,718 & 0,445 & 0,611 & 0,622 & 0,061 & 0,100 \\
\hline & $X_{2}$ & Escore acesso à empresa & 0,748 & 0,517 & 0,645 & 0,658 & 0,057 & 0,088 \\
\hline & $x_{3}$ & $\begin{array}{c}\text { Escore confiabilidade nos } \\
\text { serviços }\end{array}$ & 0,721 & 0,513 & 0,614 & 0,631 & 0,053 & 0,087 \\
\hline & $\mathrm{X}_{4}$ & Valor & 0,497 & 0,215 & 0,345 & 0,354 & 0,066 & 0,190 \\
\hline & $X_{5}$ & Satisfação & 0,716 & 0,498 & 0,614 & 0,622 & 0,059 & 0,096 \\
\hline & $X_{6}$ & Confiança & 0,681 & 0,461 & 0,602 & 0,612 & 0,053 & 0,089 \\
\hline & $X_{7}$ & Fidelidade & 0,584 & 0,237 & 0,384 & 0,388 & 0,073 & 0,189 \\
\hline \multirow{3}{*}{ II } & $\mathrm{V}_{1}$ & Q de Tobin & 1,477 & 0,882 & 1,112 & 1,098 & 0,158 & 0,142 \\
\hline & $V_{2}$ & Enterprise Value/Ebitda & 12,781 & 2,674 & 6,845 & 6,416 & 2,285 & 0,334 \\
\hline & $V_{3}$ & Capitalização de Mercado & 0,760 & 0,106 & 0,380 & 0,379 & 0,173 & 0,455 \\
\hline \multirow{5}{*}{ III } & $\mathrm{D}_{1}$ & Ebitda / Receita & 0,301 & 0,048 & 0,183 & 0,194 & 0,068 & 0,369 \\
\hline & $\mathrm{D}_{2}$ & ROE & 0,361 & $-0,049$ & 0,128 & 0,134 & 0,086 & 0,668 \\
\hline & $\mathrm{D}_{3}$ & ROA & 0,123 & $-0,012$ & 0,036 & 0,032 & 0,028 & 0,769 \\
\hline & $\mathrm{D}_{4}$ & $\mathrm{GC} / \mathrm{AT}$ & 0,198 & $-0,081$ & 0,062 & 0,062 & 0,057 & 0,915 \\
\hline & $D_{5}$ & EVA & 0,046 & $-0,114$ & $-0,039$ & $-0,040$ & 0,039 & $-0,994$ \\
\hline
\end{tabular}

Nota: Máx. = Máximo; Mín. = Mínino; I = Índice ANEEL de Satisfação do Consumidor; II = Indicadores de Valoração; III = Indicadores de Desempenho.

Fonte: elaborado pelos autores.

O coeficiente de variação, de acordo com Fávero e Belfiore (2017) permite medir a homogeneidade em relação às médias, evidenciando o risco relativo das variações. A Tabela 2 indica que as variáveis possuem valores muito próximos, sendo as de Valor $\left(\mathrm{X}_{4}\right)$ e Fidelidade $\left(\mathrm{X}_{7}\right)$ as maiores, com coeficiente de 0,20 aproximadamente. Esta proximidade entre todos os coeficientes calculados pode estar associada ao fato das variáveis medirem a percepção dos consumidores e pela possibilidade de serem resumidas em um único índice.

Verifica-se que a variável Enterprise Value/Ebitda $\left(\mathrm{V}_{2}\right)$ possui os valores mais elevados no grupo de indicadores de valoração. Considerando que a variável calcula quantos períodos são necessários para que a geração de caixa atinja o valor atual da empresa, pode-se pressupor que são necessários maiores períodos para que a geração de caixa atinja o valor atual, devido aos impactos da MP no 579/2012 e da Lei 12.783/2013 (BRASIL, 2013), visto que tal medida reduziu a tarifa energética e indiretamente elevou o custo ao utilizar energia térmica para auxiliar no suprimento de demandas, as quais surgiram principalmente em função da crise híbrida vivenciada pelo setor nos anos de 2013 e 2014 (TELES, 2015; 
TUNDISI, 2008). Referente ao coeficiente de variação, a variável de Capitalização de Mercado $\left(V_{3}\right)$ foi a que apresentou maior oscilação entre as observações realizadas.

As variáveis de desempenho possuem coeficientes de variação mais dispersados. Nota-se que o maior coeficiente é de 0,915 referente à variável Ebitda / Ativo Operacional $\left(D_{4}\right)$, que mede a rentabilidade do ativo operacional ajustado. $O$ menor coeficiente de variação é $-0,994$ referente à variável $D_{5}$ que corresponde ao EVA, variável que mede a diferença entre o retorno do capital da empresa e o custo desse capital, ou seja, permite ao acionista avaliar se o investimento lhe fornece ganhos reais. Como o período utilizado para o estudo é de 2010 a 2016, um provável motivo para tal variação negativa, é o impacto da Medida Provisória № 579 de 2012, que trouxe incertezas às distribuidoras de energia elétrica, bem como aos acionistas e consumidores.

Em 2013 a MP no 579/2012 foi instrumentalizada na Lei no 12.783/2013 (BRASIL, 2013). A intenção da MP no 579, e da Lei que a converteu, era de estabelecer redução de aproximadamente $20 \%$ na tarifa aos consumidores. Entretanto, outros fatores como a falta de chuvas e a necessidade de contratação de energia alternativa com custo mais elevado, como as de usinas térmicas, ocasionou prejuízos às companhias energéticas, visto que precisavam manter suas tarifas mais baixas, ainda com as adversidades.

\subsection{ANÁLISE FATORIAL EXPLORATÓRIA (AFE)}

Na AFE foi utilizado o método de rotação Varimax, à medida que Fávero e Belfiore (2017) argumentam que este método minimiza o número de variáveis com altas cargas em um fator, tornando a análise dos fatores mais simplificada. É realizada também a análise das matrizes de correlação de Pearson e anti-imagem, o teste de KMO e de esfericidade de Bartlett e a verificação da variância total explicada.

A partir da matriz de correlação de Pearson foi identificado que todas as associações foram maiores que 0,30 e de alta significância, ou seja, o $p$-valor $<0,05$ informa que todas as relações possíveis deram altamente significativas. Na Tabela 3 são mostrados os resultados dos testes de KMO e de esfericidade de Bartlett.

Tabela 3 - Teste de KMO e de Esfericidade de Bartlett - Variável da Pesquisa

\begin{tabular}{|c|c|c|}
\hline \multicolumn{2}{|c|}{ Medida Kaiser-Meyer-Olkin de adequação de amostragem. } & 0,830 \\
\hline \multirow{3}{*}{$\begin{array}{c}\text { Teste de esfericidade de Bartlett } \\
\text { Variável IASC }\end{array}$} & Qui-quadrado aprox. & 967,238 \\
\hline & $\mathrm{df}$ & 21 \\
\hline & p-valor & 0,000 \\
\hline \multicolumn{2}{|c|}{ Medida Kaiser-Meyer-Olkin de adequação de amostragem. } & 0,560 \\
\hline \multirow{3}{*}{$\begin{array}{l}\text { Teste de esfericidade de Bartlett } \\
\text { Indicadores de Valoração }\end{array}$} & Qui-quadrado aprox. & 132,828 \\
\hline & $\mathrm{df}$ & 3 \\
\hline & p-valor & 0,000 \\
\hline \multicolumn{2}{|c|}{ Medida Kaiser-Meyer-Olkin de adequação de amostragem. } & 0,689 \\
\hline \multirow{3}{*}{$\begin{array}{l}\text { Teste de esfericidade de Bartlett } \\
\text { Variáveis de Desempenho }\end{array}$} & Qui-quadrado aprox. & 307,929 \\
\hline & df & 10 \\
\hline & p-valor & 0,000 \\
\hline
\end{tabular}

Fonte: elaborado pelos autores.

Os resultados indicaram que a utilização da técnica AFE para a variável IASC é adequada, visto que o resultado de KMO está próximo a 1. De acordo com Fávero e Belfiore (2017), os valores entre 0,80 e 0,90 para a estatística KMO indicam uma boa análise fatorial. Já para as variáveis de valoração a utilização da AFE não é muito adequada para as variáveis

RGO - Revista Gestão Organizacional, Chapecó, v. 13, n. 2, p. 104-122, maio/ago. 2020. 
de valoração, visto que o resultado do KMO foi de 0,560. Por fim, referente as variáveis de desempenho é possível comprovar que a utilização da técnica é razoavelmente adequada, visto que o resultado é de 0,689. De acordo com Fávero e Belfiore (2017) os valores entre 0,60 e 0,70 para a estatística KMO indicam uma análise fatorial razoável.

O nível de significância dos testes de esfericidade de Bartlett conduz à rejeição da hipótese de a matriz de correlações é identidade, o que reforça que há correlações significantes entre as variáveis. Na Tabela 4 é apresentada a matriz anti-imagem das variáveis da pesquisa.

Tabela 4 - Matriz anti-imagem - variáveis de índices amplos

\begin{tabular}{|c|c|c|c|c|c|c|c|}
\hline Variáveis IASC & $\mathbf{X 1}$ & $\mathrm{X2}$ & $\mathrm{X3}$ & $\mathrm{X4}$ & $\mathrm{X5}$ & $\mathrm{x6}$ & $\mathrm{x7}$ \\
\hline \multirow{7}{*}{$\begin{array}{c}\text { Covariância } \\
\text { Anti- } \\
\text { imagem }\end{array}$} & 0,089 & $-0,039$ & 0,008 & 0,024 & 0,016 & $-0,044$ & $-0,038$ \\
\hline & $-0,039$ & 0,048 & $-0,041$ & $-0,043$ & $-0,031$ & 0,020 & 0,053 \\
\hline & 0,008 & $-0,041$ & 0,069 & 0,035 & 0,006 & $-0,033$ & $-0,037$ \\
\hline & 0,024 & $-0,043$ & 0,035 & 0,530 & 0,002 & $-0,014$ & $-0,236$ \\
\hline & 0,016 & $-0,031$ & 0,006 & 0,002 & 0,225 & $-0,070$ & $-0,011$ \\
\hline & $-0,044$ & 0,020 & $-0,033$ & $-0,014$ & $-0,070$ & 0,134 & $-0,055$ \\
\hline & $-0,038$ & 0,053 & $-0,037$ & $-0,236$ & $-0,011$ & $-0,055$ & 0,401 \\
\hline \multirow{7}{*}{$\begin{array}{c}\text { Correlação } \\
\text { Anti- } \\
\text { imagem }\end{array}$} & $0,859^{a}$ & $-0,597$ & 0,105 & 0,112 & 0,113 & $-0,406$ & $-0,203$ \\
\hline & $-0,597$ & $0,745^{a}$ & $-0,711$ & $-0,267$ & $-0,300$ & 0,248 & 0,379 \\
\hline & 0,105 & $-0,711$ & $0,836^{\mathrm{a}}$ & 0,183 & 0,049 & $-0,340$ & $-0,225$ \\
\hline & 0,112 & $-0,267$ & 0,183 & $0,804^{a}$ & 0,007 & $-0,054$ & $-0,511$ \\
\hline & 0,113 & $-0,300$ & 0,049 & 0,007 & $0,923^{\mathrm{a}}$ & $-0,402$ & $-0,037$ \\
\hline & $-0,406$ & 0,248 & $-0,340$ & $-0,054$ & $-0,402$ & $0,865^{a}$ & $-0,238$ \\
\hline & $-0,203$ & 0,379 & $-0,225$ & $-0,511$ & $-0,037$ & $-0,238$ & $0,758^{a}$ \\
\hline \multicolumn{2}{|c|}{ Variáveis de Valoração } & \multicolumn{2}{|c|}{ V1 } & \multicolumn{2}{|c|}{ V2 } & \multicolumn{2}{|c|}{ V3 } \\
\hline \multirow{3}{*}{$\begin{array}{c}\text { Covariância } \\
\text { anti- } \\
\text { imagem }\end{array}$} & V1 & \multicolumn{2}{|c|}{0,331} & \multicolumn{2}{|c|}{$-0,185$} & \multicolumn{2}{|c|}{$-0,270$} \\
\hline & V2 & \multicolumn{2}{|c|}{$-0,185$} & \multicolumn{2}{|c|}{0,786} & \multicolumn{2}{|c|}{0,050} \\
\hline & V3 & \multicolumn{2}{|c|}{$-0,270$} & \multicolumn{2}{|c|}{0,050} & \multicolumn{2}{|c|}{0,378} \\
\hline \multirow{3}{*}{$\begin{array}{c}\text { Correlação } \\
\text { anti- } \\
\text { imagem }\end{array}$} & V1 & \multicolumn{2}{|c|}{$0,536^{a}$} & \multicolumn{2}{|c|}{$-0,362$} & \multicolumn{2}{|c|}{$-0,763$} \\
\hline & V2 & \multicolumn{2}{|c|}{$-0,362$} & \multicolumn{2}{|c|}{$0,684^{a}$} & \multicolumn{2}{|c|}{0,091} \\
\hline & V3 & \multicolumn{2}{|c|}{$-0,763$} & & & & \\
\hline Variáveis de & Desempenho & D1 & D2 & & & D4 & D5 \\
\hline & D1 & 0,368 & 0,076 & & & $-0,022$ & $-0,024$ \\
\hline & D2 & 0,076 & 0,393 & & & 0,048 & 0,037 \\
\hline Covariancla & D3 & $-0,138$ & $-0,157$ & & & $-0,127$ & $-0,125$ \\
\hline & D4 & $-0,022$ & 0,048 & & & 0,596 & 0,218 \\
\hline & D5 & $-0,024$ & 0,037 & & & 0,218 & 0,563 \\
\hline & D1 & $0,778^{a}$ & 0,200 & & & $-0,047$ & $-0,053$ \\
\hline & D2 & 0,200 & $0,718^{a}$ & & & 0,099 & 0,078 \\
\hline Correlaçao & D3 & $-0,576$ & $-0,634$ & & & $-0,419$ & $-0,423$ \\
\hline & D4 & $-0,047$ & 0,099 & & & $0,664^{a}$ & 0,376 \\
\hline & D5 & $-0,053$ & 0,078 & & & 0,376 & $0,697^{a}$ \\
\hline
\end{tabular}

Fonte: elaborado pelos autores.

Nota-se que na matriz anti-imagem de correlações, os valores da diagonal principal (conhecida como MSA - Medida de Adequação da Amostra) são superiores a 0,50, o que confirma novamente a adequação da análise fatorial para o modelo de associação proposto. Caso algum valor estivesse abaixo de 0,50 , a variável específica poderia não se ajustar à estrutura definida pelas outras variáveis, devendo ser avaliada a sua eliminação da amostra. 
Após a verificar a congruência dos indicadores, foi analisada a extração de fatores e a variância total explicada das variáveis exploradas nesta pesquisa, conforme Tabela 5.

Tabela 5 - Variância total explicada - variáveis da pesquisa

\begin{tabular}{|c|c|c|c|c|c|c|}
\hline \multirow{2}{*}{$\begin{array}{l}\text { Componente } \\
\text { IASC }\end{array}$} & \multicolumn{3}{|c|}{ Valores próprios iniciais } & \multicolumn{3}{|c|}{$\begin{array}{l}\text { Somas de extração de carregamentos } \\
\text { ao quadrado }\end{array}$} \\
\hline & Total & $\begin{array}{c}\text { \% de } \\
\text { variância }\end{array}$ & $\begin{array}{c}\% \\
\text { cumulativa }\end{array}$ & Total & $\begin{array}{c}\text { \% de } \\
\text { variância }\end{array}$ & $\begin{array}{c}\% \\
\text { cumulativa }\end{array}$ \\
\hline 1 & 5,183 & 74,042 & 74,042 & \multirow{7}{*}{5,183} & \multirow{7}{*}{74,042} & \multirow{7}{*}{74,042} \\
\hline 2 & 0,952 & 13,602 & 87,643 & & & \\
\hline 3 & 0,401 & 5,728 & 93,371 & & & \\
\hline 4 & 0,221 & 3,156 & 96,527 & & & \\
\hline 5 & 0,127 & 1,819 & 98,346 & & & \\
\hline 6 & 0,087 & 1,249 & 99,594 & & & \\
\hline 7 & 0,028 & 0,406 & 100,000 & & & \\
\hline \multirow{2}{*}{$\begin{array}{l}\text { Componente } \\
\text { Variáveis de } \\
\text { Valoração }\end{array}$} & \multicolumn{3}{|c|}{ Valores próprios iniciais } & \multicolumn{3}{|c|}{$\begin{array}{l}\text { Somas de extração de carregamentos } \\
\text { ao quadrado }\end{array}$} \\
\hline & Total & $\begin{array}{c}\text { \% de } \\
\text { variância }\end{array}$ & $\begin{array}{c}\% \\
\text { cumulativa }\end{array}$ & Total & $\begin{array}{c}\text { \% de } \\
\text { variância }\end{array}$ & $\begin{array}{c}\% \\
\text { cumulativa }\end{array}$ \\
\hline 1 & 2,062 & 68,745 & 68,745 & \multirow{3}{*}{2,062} & \multirow{3}{*}{68,745} & \multirow{3}{*}{68,745} \\
\hline 2 & 0,742 & 24,731 & 93,476 & & & \\
\hline 3 & 0,196 & 6,524 & 100,000 & & & \\
\hline \multirow{2}{*}{$\begin{array}{l}\text { Componente } \\
\text { Variáveis de } \\
\text { Desempenho }\end{array}$} & \multicolumn{3}{|c|}{ Valores próprios iniciais } & \multicolumn{3}{|c|}{$\begin{array}{l}\text { Somas de extração de carregamentos } \\
\text { ao quadrado }\end{array}$} \\
\hline & Total & $\begin{array}{c}\text { \% de } \\
\text { variância }\end{array}$ & $\begin{array}{c}\% \\
\text { cumulativa }\end{array}$ & Total & $\begin{array}{c}\text { \% de } \\
\text { variância }\end{array}$ & $\begin{array}{c}\% \\
\text { cumulativa }\end{array}$ \\
\hline 1 & 3,074 & 61,471 & 61,471 & \multirow{5}{*}{3,074} & \multirow{5}{*}{61,471} & \multirow{5}{*}{61,471} \\
\hline 2 & 0,930 & 18,598 & 80,068 & & & \\
\hline 3 & 0,514 & 10,272 & 90,341 & & & \\
\hline 4 & 0,373 & 7,467 & 97,808 & & & \\
\hline 5 & 0,110 & 2,192 & 100,000 & & & \\
\hline
\end{tabular}

Identifica-se que a combinação linear das variáveis do IASC são altamente correlacionadas. Essas variáveis podem ser combinadas formando um único fator que explica $74,04 \%$ da variância dos dados originais. Esses resultados sugerem que a mensuração dos indicadores do IASC são redundantes com a maioria deles medindo praticamente a mesma dimensão, ainda que o conteúdo dos instrumentos de pesquisa se mostrem diferentes. Quanto as variáveis de valoração de empresa, observa-se que a combinação linear das variáveis é correlacionada, essas variáveis podem ser combinadas formando um único fator que explicará $68,75 \%$ da variância dos dados originais.

A análise das variáveis de desempenho indicou a existência de alta correlação de Pearson em pares, com associações maiores que 0,30 e significativas. Somente a variável $D_{5}$ que corresponde ao EVA, apresentou correlação não significante. Verifica-se que a combinação linear das variáveis é correlacionada, essas variáveis podem ser combinadas formando um único fator que explicará $61,47 \%$ da variância dos dados originais das variáveis de desempenho. Em suma, os resultados detalhados na Tabela 5 evidenciam que as variáveis do IASC, as variáveis de valoração e as variáveis de desempenho formam fatores únicos. 


\subsection{CORRELAÇÃO ENTRE OS FATORES DO IASC, DE VALORAÇÃO E DE DESEMPENHO}

Após serem formados os fatores dos grupos de variáveis do IASC, de valoração e de desempenho, as pontuações de cada observação foram salvas com intuito de verificar a correlação existente entre esses fatores. Segundo Fávero e Belfiore (2017), os fatores criados pela análise fatorial exploratória podem ser empregados em testes multivariados, substituindo as variáveis originais. Para tanto, foi aplicado também o teste de KolmogorovSmirnov que apontou a não normalidade dos dados. Perante isso, para realizar a análise de correlações é utilizada as matrizes de correlações de bivariáveis de Spearman, uma análise não paramétrica que não necessita de dados robustos (Field, 2009).

Por meio da matriz de correlações de Spearman é verificada a força da associação entre as variáveis elencadas na investigação, que podem variar entre - 1 e 1 . Quanto mais próximo de 1 , mais forte a associação, quanto mais próximo de -1 , mais inversa é a associação. A Tabela 6 apresenta a Matriz de Correlações de Spearman para as variáveis da pesquisa, com vista a identificar as possíveis associações existentes.

Tabela 6 - Matriz de correlações de Spearman

\begin{tabular}{|c|c|c|c|c|}
\hline \multicolumn{3}{|c|}{ Fator do IASC x Fator de Valoração } & $\begin{array}{l}\text { Fator do } \\
\text { lasc }\end{array}$ & $\begin{array}{l}\text { Fator de } \\
\text { Valoração }\end{array}$ \\
\hline \multirow{4}{*}{ Spearman } & \multirow{2}{*}{ Fator do lasc } & Correlações & 1,000 & 0,053 \\
\hline & & p-valor & & 0,577 \\
\hline & \multirow{2}{*}{$\begin{array}{l}\text { Fator de } \\
\text { Valoração }\end{array}$} & Correlações & 0,053 & 1,000 \\
\hline & & p-valor & \multicolumn{2}{|c|}{0,577} \\
\hline \multicolumn{3}{|c|}{ Fator do IASC x Fator de Desempenho } & $\begin{array}{c}\text { Fator do } \\
\text { lasc }\end{array}$ & $\begin{array}{c}\text { Fator de } \\
\text { Desempenho }\end{array}$ \\
\hline \multirow{4}{*}{ Spearman } & \multirow{2}{*}{ Fator do lasc } & Correlações & 1,000 & $0,222^{*}$ \\
\hline & & p-valor & & 0,018 \\
\hline & \multirow{2}{*}{$\begin{array}{c}\text { Fator de } \\
\text { Desempenho }\end{array}$} & Correlações & $0,222^{*}$ & 1,000 \\
\hline & & p-valor & \multicolumn{2}{|c|}{0,018} \\
\hline \multicolumn{3}{|c|}{ Fator de Desempenho x Fator de Valoração } & $\begin{array}{c}\text { Fator de } \\
\text { Valoração }\end{array}$ & $\begin{array}{c}\text { Fator de } \\
\text { Desempenho }\end{array}$ \\
\hline \multirow{4}{*}{ Spearman } & \multirow{2}{*}{$\begin{array}{c}\text { Fator de } \\
\text { Valoração }\end{array}$} & Correlações & 1,000 & $0,631 * *$ \\
\hline & & p-valor & & 0,000 \\
\hline & \multirow{2}{*}{$\begin{array}{c}\text { Fator de } \\
\text { Desempenho }\end{array}$} & Correlações & $0,631 * *$ & 1,000 \\
\hline & & p-valor & \multicolumn{2}{|c|}{0,000} \\
\hline
\end{tabular}

Nota: *Significante ao nível de $5 \%$. **Significante ao nível de $1 \%$.

Fonte: elaborado pelos autores.

A partir dos resultados apresentados na Tabela 6 é possível observar que a correlação entre o fator do IASC e o fator de Valoração não indicou associação significante. $O$ p-valor calculado é de 0,577 , superior a $5 \%$, fato que culmina para a não possibilidade de confirmação da hipótese que defende que existe associação entre o Índice ANEEL de Satisfação do Consumidor e os indicadores de valoração de empresas distribuidoras de energia elétrica listadas na $[B]^{3}$. Com os resultados obtidos, pode-se inferir que não há associação entre a qualidade dos serviços prestados e a valoração de mercado das distribuidoras de energia elétrica, resultado convergente com a pesquisa de Pignanelli e Csillag (2008). Os achados levam a crer que outros fatores se associam de forma mais contundente a variação de mercado dessas empresas do que o IASC, como cenário econômico, resultados históricos e interferência da regulação. Adicionalmente, esses 
resultados sugerem que os índices de satisfação são sensíveis aos fundamentos das companhias do setor elétrico e não ao valor percebido das ações das empresas pelo mercado acionário.

Por outro lado, ao nível de $5 \%$, é possível confirmar a hipótese de que existe associação significante entre o Índice ANEEL de Satisfação do Consumidor e de desempenho de empresas distribuidoras de energia elétrica listadas $n a[B]^{3}$. Esta decisão está sustentada na correlação entre o fator do IASC e o fator de Desempenho que apresentou um $p$-valor de 0,018 , inferior a $5 \%$. Desse modo, os resultados se alinham aos achados de Pignanelli e Csillag (2008), Almeida et al. (2010) e Catapan et al. (2017), de que as empresas com maiores indicadores de qualidade apresentam melhor desempenho financeiro advindos de suas atividades. Nesse contexto, os indicadores de qualidade podem traduzir a eficiência da organização em aproveitar seus recursos na prestação de serviços ao cliente, que por sua vez, se associaria ao desempenho financeiro.

Tais achados corroboram com Albuquerque, Carvalho e Bonizio (2014) que acreditam que a qualidade dos serviços prestados pelas organizações reflete não só na competitividade da organização, mas também em seu desempenho financeiro o que favorece a continuidade organizacional. Catapan et al. (2017) também destaca a importância de direcionar olhares para a satisfação dos consumidores, pois esta é capaz de definir os rumos de uma organização. Tais discussões encontram respaldo nos resultados aqui expostos.

Embora não seja objeto do estudo o exame de associação entre os fatores de desempenho e valoração, a correlação entre estes apresentou a existência de correlação com p-valor de 0,000. Nesse sentido, apesar de não haver associação direta entre o IASC e o fator de valoração, há associação entre a valoração e o desempenho. Assim, de forma indireta ao investir na qualidade dos serviços prestados pode-se maximizar o desempenho da empresa maximizar positivamente a valoração de mercado da organização. Além disso, pode-se sugerir que o desempenho e a valoração de companhias do setor elétrico estão associados à satisfação do consumidor.

\section{CONSIDERAÇÕES FINAIS}

A presente pesquisa teve como objetivo verificar a associação entre o Índice ANEEL de Satisfação do Consumidor, os indicadores de valoração e de desempenho de companhias distribuidoras de energia elétrica listadas na Brasil Bolsa Balcão $[B]^{3}$. Os resultados mostraram não haver associação significante do IASC com os indicadores de valoração das empresas analisadas. Por outro lado, foi identificada associação positiva e significante das variáveis que consubstanciam o IASC com o desempenho financeiro das empresas. Por fim, houve alta associação entre o fator de desempenho com o de valoração.

Os achados da pesquisa contribuem com a literatura à medida que sugerem que a qualidade dos serviços prestados pode significar melhor desempenho empresarial, pois a empresa tende a ser mais eficiente no uso de seus recursos com intuito de obter maior lucratividade. Esses resultados são particularmente importante, visto que Albuquerque, Carvalho e Bonizio (2014) reconhecem que ao focar na qualidade as empresas podem apresentar um diferencial competitivo perante seus concorrentes.

Em termos de valoração de mercado não foi encontrada associação significante. Com isso, o estudo fornece oportunidades de investigações alternativas que destaquem outros fatores como aspectos especulativos, de regulação e econômicos, que podem estar correlacionados com a valoração das empresas analisadas. Ademais, estudos futuros podem demandar atenção em compreender se o mercado acionário direciona olhares para a 
qualidade dos serviços prestados ou se o foco está estritiamente no desempenho financeiro. Tal proposição de estudos futuros encontra respaldo na terceira análise realizada, a qual indicou alta correlação entre a valoração de mercado com o desempenho financeiro das empresas analisadas.

Os resultados ainda contribuem com a literatura empírica, pois os trabalhos que abordam esses aspectos do setor elétrico ainda são precedentes. Dessa forma, esta pesquisa contribui para o campo acadêmico, à medida que reconhece a necessidade de integração de variáveis não quantitativas e quantitativas para o desenvolvimento das organizações, em especial setores que recebem o intervencionismo do Estado de modo direto no que incumbe fomento, regulação e fiscalização (SANTOS, 2010). Os resultados também indicam a possível existência de redundância entre as variáveis utilizadas no questionário de satisfação da ANEEL, visto que tais variáveis tendem a mensurar os mesmos aspectos sem capturar as diferentes nuances da qualidade do atendimento prestado aos usuários.

As limitações da pesquisa pairam no número restrito de empresas do setor elétrico que possuem dados disponíveis para serem analisados e pela carência de estudos teóricos para oferecer suporte às discussões apresentadas. Estudos futuros podem utilizar outras técnicas de análise de dados para ampliar as discussões acerca da temática. Ademais, destaca-se que uma oportunidade de pesquisa é investigar o possível papel interveniente de indicadores de desempenho na relação entre o IASC e os índices de valoração das companhias do setor elétrico.

\section{REFERÊNCIAS}

ALBUQUERQUE, A. A. DE; CARVALHO, F. L. DE; BONIZIO, R. C. Qualidade e rentabilidade : um estudo aplicado nas distribuidoras de energia elétrica brasileiras. RIC - Revista de Informação Contábil, v. 8, n. 3, p. 139-156, 2014.

ALMEIDA, R. S.; COLARES, A. C. V.; LAMOUNIER, W. M.; MÁRIO, P. DO C. Qualidade do serviço, satisfação do consumidor e desempenho financeiro das empresas distribuidoras de energia elétrica brasileiras. In: Congresso Brasileiro de Custos, 17., Belo Horizonte. Anais... [...]. Belo Horizonte: ABC, 2010. Disponível em: https://anaiscbc.emnuvens.com.br/anais/article/view/824. Acesso em: 16 mai. 2020.

ANEEL - Agêngia Nacional de Energia Elétrica. Metodologia. 2015. Disponível em: http://www.aneel.gov.br/metodologia-IASC/-/asset_publisher/ri7lpR3R2YkT/content/o-quee-/655804 ?inheritRedirect $=$ false \& redirect=http\%3A\%2F\%2Fwww.aneel.gov.br\%2Fmetodologia-IASC\%3Fp_p_id \%3D 101_INSTANCE_ri7IpR3R2YkT\%26p_p_lifecycle\%3D0\%26p_p_state\%3Dnormal\%26p_p_mod e\%3Dview\%26p_p_col_id\%3Dcolumn-2\%26p_p_col_count\%3D1. Acesso em: 01 ago. 2018.

ANEEL - Agêngia Nacional de Energia Elétrica. Índice ANEEL de Satisfação do Consumidor (IASC). 2015. Disponível em: http://www.aneel.gov.br/indice-aneel-satisfacao-consumidor. Acesso em: 01 ago. 2018.

ANEEL - Agêngia Nacional de Energia Elétrica. Programa de Eficiência Energética. 2015. Disponível em: http://www.aneel.gov.br/programa-eficiencia-energetica. Acesso em: 01 ago. 2018. 
ANEEL - Agêngia Nacional de Energia Elétrica. Módulo 8-Qualidade da Energia Elétrica. 2017. Disponível em:

http://www.aneel.gov.br/documents/656827/14866914/M\%C3\%B3dulo8_Revisao_8/9c78cf ab-a7d7-4066-b6ba-cfbda3058d19. Acesso em: 01 ago. 2018.

ANEEL - Agêngia Nacional de Energia Elétrica. Relatório Pesquisa IASC 2017: Brasil, categorias e distribuidoras. 2017. Disponível em:

http://www.aneel.gov.br/documents/655804/15358423/relatorio_IASC_2017_vs_web_final _compac.pdf/c1b2ad51-aeed-1d49-f3b8-3439f2b1b6a3. Acesso em: 01 ago. 2018.

ANEEL - Agêngia Nacional de Energia Elétrica. A ANEEL. 2018. Disponível em: http://www.aneel.gov.br/a-aneel. Acesso em: 01 ago. 2018.

ASSAF NETO. Curso de administração financeira. São Paulo: Atlas, 2008.

BODIE, Z.; KANE, A.; MARCUS, A. Fundamentos de investimentos. AMGH Editora, 2014.

BRASIL. LEI № 9.427 DE 26 DE DEZEMBRO DE 1996. Institui a Agência Nacional de Energia Elétrica - ANEEL, disciplina o regime das concessões de serviços públicos de energia elétrica e dá outras providências, Brasília, DF, dez. 1996. Disponível em:

https://guiadamonografia.com.br/como-fazer-citacao-de-lei/. Acesso em: 01 ago. 2018.

BRASIL. LEI № 12.783 DE 11 DE JANEIRO DE 2013. Dispõe sobre as concessões de geração, transmissão e distribuição de energia elétrica, sobre a redução dos encargos setoriais e sobre a modicidade tarifária [...]. Brasília, DF, jan. 2013 . Disponível em:

http://www.planalto.gov.br/ccivil_03/_Ato2011-2014/2013/Lei/L12783.htm. Acesso em: 01 ago. 2018.

BRASIL. MEDIDA PROVISÓRIA № 579 DE 11 DE SETEMBRO DE 2012. Dispõe sobre as concessões de geração, transmissão e distribuição de energia elétrica, sobre a redução dos encargos setoriais, sobre a modicidade tarifária, e dá outras providências, Brasília, DF, set. 2012. Disponível em: http://www.planalto.gov.br/ccivil_03/_Ato20112014/2012/Mpv/579.htm. Acesso em: 01 ago. 2018.

CATAPAN, A. M.; YAMAKAWA, E. K.; SIEBERT, L. C.; AOKI, A. R.; LIMA, E. P. Residential Customer Satisfaction Performance Assessment Model for Electricity Service for a Power Utility in Brazil. In: Portland International Conference on Management of Engineering and Technology (PICMET), Portland. Anais...[...] Portland: 2017, Portland States University. Disponível em: https://ieeexplore.ieee.org/document/8125464. Acesso em: 16 mai. 2020.

FAMÁ, R.; BARROS, L. A. B. C. Q de Tobin e seu uso em finanças: aspectos metodológicos e conceituais. Caderno de Pesquisas em Administração, v. 7, n. 4, p. 27-43, 2000.

FÁVERO, L. P.; BELFIORE, P. Manual de análise de dados - Estatística e Modelagem Multivariada com Exce $^{\circledR}$, SPSS $^{\circledR}$ e Stata ${ }^{\circledR}$. Rio de Janeiro: Elsevier, 2017.

FIELD, A. Descobrindo a Estatística usando o SPSS. 2. ed. Porto Alegre: Artmed, 2009. 
GASPARETTO, V.; LOPES, I. F.; SANTOS, V.; SCHNORRENBERGER, D. Evidenciação dos Fatores de Riscos de Companhias Elétricas Diante de uma Crise Hídrica. In: USP International Conference in Accounting, 18., 2018, São Paulo. Anais...[...]. São Paulo: USP, 2018. Disponível em:

https://congressousp.fipecafi.org/anais/Anais2018/ArtigosDownload/796.pdf. Acesso em: 16 mai. 2020.

GOLDENBERG, J.; PRADO, L. T. S. Reforma e crise do setor elétrico no período FHC. Tempo Social, v. 15, n. 2, p. 219-235, 2003. DOI: https://doi.org/10.1590/S010320702003000200009

GUIMARÃES, F. L. A crise da energia elétrica e o seu custo. Revista USP, v. 104, p. 83-90, 2015.

HAIR JR, J. F.; BLACK, W. C.; BABIN, B. J.; ANDERSON, R. E.; TATHAM, R. L. Análise multivariada de dados. Porto Alegre: Bookman, 2009.

LIMA, M. A. A. Uma nova metodologia para análise da qualidade da energia elétrica sob condições de ocorrência de múltiplos distúrbios. 2013. Tese (Doutorado em Ciências Contábeis) - Programa de Pós-Graduação em Ciências Contábeis, Universidade de São Paulo, São Carlos, SP, 2013.

MARTINS, E. Avaliando a Empresa (I) e (II). Temática Contábil. Bol 10 e 11, 1998.

MARINHO, A.; RESENDE, M. Service Quality in Electricity Distribution in Brazil: A Malmquist Approach. Annals of Public and Cooperative Economics, p. 1-26, 2016. DOI: https://doi.org/10.1111/apce.12241

NEVES JUNIOR, I. J.; BATISTA, C. P. Estudo empírico do desempenho das empresas de distribuição de energia elétrica segundo o Ebitda. Revista de Contabilidade do Mestrado em Ciências Contábeis da UERJ, v. 10, n. 2, 2005, p. 27-40. DOI: https://doi.org/10.12979/rcmccuerj.v10i2.5593

PÉREZ, J. P. G. Qualidade do serviço de distribuição de energia elétrica: indicadores, limites, compensações e incentivos regulatórios. 2017. Tese (Doutorado em Ciências Contábeis) - Programa de Pós-Graduação em Ciências Contábeis, Universidade de São Paulo, São Paulo, SP, 2017.

PIGNANELLI, A.; CSILLAG, J. M. The Impact of Quality Management on Profitability: An Empirical Study. Journal of Operations and Supply Chain Management, v. 1, n. 1, p. 66-77, 2008. DOI: http://dx.doi.org/10.12660/joscmv1n1p66-77

RESENDE, M.; CARDOSO, V. Mapping service quality in electricity distribution: an exploratory study of Brazil. Utilities Policy, v. 56, p. 41-52, 2019. DOI:

https://doi.org/10.1016/j.jup.2018.08.009 
SANTANA, L.; LIMA, F. G. EBITDA: uma Análise de Correlação com os Retornos Totais aos Acionistas no Mercado de Capitais Brasileiro. In: USP International Conference in Accounting, 4., 2004, São Paulo. Anais...[...]. São Paulo: USP, 2004. Disponível em: https://congressousp.fipecafi.org/anais/artigos42004/374.pdf. Acesso em: 16 mai. 2020.

SANTOS, L. A. Condições de Concorrência em Setores Regulados: O Caso do Serviço Telefônico Fixo Comutado. Caderno Virtual, v. 1, n. 21, p. 1-273, 2010.

TELES, L. F. A. Crise hídrica destaca o papel estratégico da sustentabilidade nas organizações. 2015. Disponível em:

http://www.fdc.org.br/blogespacodialogo/Lists/Postagens/Post.aspx?ID=417. Acesso em: 16 mai. 2020.

TUNDISI, J. G. Recursos hídricos no futuro: problemas e soluções. Estudos Avançados, v. 22, n. 63, p. 7-16, 2008. DOI: http://dx.doi.org/10.1590/S0103-40142008000200002.

WALVIS, A.; GONÇALVES, E. D. L. Avaliação das reformas recentes no setor elétrico brasileiro e sua relação com o desenvolvimento do mercado livre de energia. 2014.

Dissertação (Mestrado em Finanças e Economia Empresarial) - Programa de Mestrado em Finanças e Economia Empresarial, Fundação Getúlio Vargas, Rio de Janeiro, RJ, 2014. 